\title{
On fuzzy $\alpha$-continous multifunctions
}

Khaled M. A. Al-Hamadi and S. B. Nimse 


\title{
ON FUZZY $\alpha$-CONTINUOUS MULTIFUNCTIONS
}

\author{
KHALED M. A. AL-HAMADI AND S. B. NIMSE
}

Received 24 August, 2006

\begin{abstract}
In this paper we use fuzzy $\alpha$-sets in order to obtain certain characterizations and properties of upper (or lower) fuzzy $\alpha$-continuous multifunctions.
\end{abstract}

2000 Mathematics Subject Classification: 54A40

Keywords: fuzzy $\alpha$-open, fuzzy $\alpha$-continuous, fuzzy multifunction

\section{INTRODUCTION}

In 1968 Chang [3] introduced fuzzy topological spaces by using fuzzy sets [12]. Since then several workers have contributed to this area: various types of functions play a significant role in the theory of classical point set topology. A great number of papers dealing with such functions have appeared, and a good many of them have been extended to the setting of multifunctions.

In 1988 Neubrunn [6] and others [9]] introduced the concept of $\alpha$-continuous multifunctions. Njasted [7] and Mashhour [4] introduced $\alpha$-open ( $\alpha$-closed) sets, respectively. Bin Shahna in [2] defined these concepts in the fuzzy setting. In this paper our purpose is to define upper (lower) fuzzy $\alpha$-continuous multifunctions and to obtain several characterizations of upper (lower) fuzzy $\alpha$-continuous multifunctions.

Fuzzy sets on a universe $X$ will be denoted by $\mu, \rho, \eta$, etc. Fuzzy points will be denoted by $x_{\epsilon}, y_{\nu}$, etc. For any fuzzy points $x_{\epsilon}$ and any fuzzy set $\mu$, we write $x_{\epsilon} \in \mu$ iff $\epsilon \leq \mu(x)$. A fuzzy point $x_{\epsilon}$ is called quasi-coincident with a fuzzy set $\rho$, denoted by $x_{\epsilon} \mathrm{q} \rho$, iff $\epsilon+\rho(x)>1$.

A fuzzy set $\mu$ is called quasi-coincident with a fuzzy set $\rho$, denoted by $\mu \mathrm{q} \rho$, iff there exists a $x \in X$ such that $\mu(x)+\rho(x)>1$. [10,11]

In this paper we use the concept of fuzzy topological space as introduced in [3]. By int $(\mu)$ and $\mathrm{cl}(\mu)$, we mean the interior of $\mu$ and the closure of $\mu$, respectively.

Let $(X, \tau)$ be a topological space in the classical sense and $(Y, \nu)$ be a fuzzy topological space. $F: X \rightarrow Y$ is called a fuzzy multifunction iff for each $x \in X, F(x)$ is a fuzzy set in $Y$. [8] 
Let $F: X \rightarrow Y$ be a fuzzy multifunction from a fuzzy topological space $X$ to a fuzzy topological space $Y$. For any fuzzy set $\mu \leq X, F^{+}(\mu)$ and $F^{-}(\mu)$ are defined by $F^{+}(\mu)=\{x \in X: F(x) \leq \mu\}, F^{-}(\mu)=\{x \in X: F(x) q \mu\}$. [5]

\section{FUZZY $\alpha$-CONTINUOUS MULTIFUNCTION}

Definition 1. Let $(X, \tau)$ be a fuzzy topological space and let $\mu \leq X$ be a fuzzy set. Then it is said that:

(i) $\mu$ is fuzzy $\alpha$-open set [2] if $\mu \leq$ int cl int $\mu$.

(ii) $\mu$ is fuzzy $\alpha$-closed set [2] if $\mu \geq \operatorname{cl}$ int $\operatorname{cl} \mu$.

(iii) $\mu$ is fuzzy semiopen set [1] if $\mu \leq \mathrm{cl}$ int $\mu$.

(iv) $\mu$ is fuzzy preopen set [2] if $\mu \leq$ int $\mathrm{cl} \mu$.

Definition 2. Let $F: X \rightarrow Y$ be a fuzzy multifunction from a fuzzy topological space $(X, \tau)$ to a fuzzy topological space $(Y, \nu)$. Then it is said that $F$ is :

(1) Upper fuzzy $\alpha$-continuous at $x_{\epsilon} \in X$ iff for each fuzzy open set $\mu$ of $Y$ containing $F\left(x_{\epsilon}\right)$, there exists a fuzzy $\alpha$-open set $\rho$ containing $x_{\epsilon}$ such that $\rho \leq F^{+}(\mu)$.

(2) Lower fuzzy $\alpha$-continuous at $x_{\epsilon} \in X$ iff for each fuzzy open set $\mu$ of $Y$ such that $x_{\epsilon} \in F^{-}(\mu)$ there exists a fuzzy $\alpha$-open set $\rho$ containing $x_{\epsilon}$ such that $\rho \leq F^{-}(\mu)$.

(3) Upper (lower) fuzzy $\alpha$-continuous iff it has this property at each point of $X$.

We know that a net $\left(x_{\epsilon_{\alpha}}^{\alpha}\right)$ in a fuzzy topological space $(X, \tau)$ is said to be eventually in the fuzzy set $\rho \leq X$ if there exists an index $\alpha_{0} \in J$ such that $x_{\epsilon_{\alpha}}^{\alpha} \in \rho$ for all $\alpha \geq \alpha_{0}$.

The following theorem states some characterizations of upper fuzzy $\alpha$-continuous multifunction.

Definition 3. A sequence $\left(x_{\epsilon_{n}}\right)$ is said to $\alpha$-converge to a point $X$ if for every fuzzy $\alpha$-open set $\mu$ containing $x_{\epsilon}$ there exists an index $n_{0}$ such that for $n \geq n_{0}, x_{\epsilon_{n}} \in \mu$. This is denoted by $x_{\epsilon_{n}} \rightarrow_{\alpha} x_{\epsilon}$.

Theorem 1. Let $F: X \rightarrow Y$ be a fuzzy multifunction from a fuzzy topological $(X, \tau)$ to a fuzzy topological space $(Y, v)$. Then the following statements are equivalent:

(i) $F$ is upper fuzzy $\alpha$-continuous.

(ii) For each $x_{\epsilon} \in X$ and for each fuzzy open set $\mu$ such that $x_{\epsilon} \in F^{+}(\mu)$ there exists a fuzzy $\alpha$-open set $\rho$ containing $x_{\epsilon}$ such that $\rho \leq F^{+}(\mu)$.

(iii) $F^{+}(\mu)$ is a fuzzy $\alpha$-open set for any fuzzy open set $\mu \leq Y$.

(iv) $F^{-}(\mu)$ is a fuzzy $\alpha$-closed set for any fuzzy open set $\mu \leq Y$.

(v) For each $x_{\epsilon} \in X$ and for each net $\left(x_{\epsilon_{\alpha}}^{\alpha}\right)$ which $\alpha$-converges to $x_{\epsilon}$ in $X$ and for each fuzzy open set $\mu \leq Y$ such that $x_{\epsilon} \in F^{+}(\mu)$, the net $\left(x_{\epsilon_{\alpha}}^{\alpha}\right)$ is eventually in $F^{+}(\mu)$. 
Proof. (i) $\Leftrightarrow$ (ii) this statement is obvious.

(i) $\Leftrightarrow$ (iii). Let $x_{\epsilon} \in F^{+}(\mu)$ and let $\mu$ be a fuzzy open set. It follows from (i) that there exists a fuzzy $\alpha$-open set $\rho_{x_{\epsilon}}$ containing $x_{\epsilon}$ such that $\rho_{x_{\epsilon}} \leq F^{+}(\mu)$. It follows that $F^{+}(\mu)=\bigvee_{x_{\epsilon} \in F^{+}(\mu)} \rho_{x_{\epsilon}}$ and hence $F^{+}(\mu)$ is fuzzy $\alpha$-open.

The converse can be shown easily.

(iii) $\Rightarrow$ (iv) Let $\mu \leq Y$ be a fuzzy open set. We have that $Y \backslash \mu$ is a fuzzy open set. From (iii), $F^{+}(Y \backslash \mu)=X \backslash F^{-}(\mu)$ is a fuzzy $\alpha$-open set. Then it is obtained that $F^{-}(\mu)$ is a fuzzy $\alpha$-closed set.

(i) $\Rightarrow(\mathrm{v})$. Let $\left(x_{\epsilon_{\alpha}}^{\alpha}\right)$ be a net which $\alpha$-converges to $x_{\epsilon}$ in $X$ and let $\mu \leq Y$ be any fuzzy open set such that $x_{\epsilon} \in F^{+}(\mu)$. Since $F$ is an upper fuzzy $\alpha$-continuous multifunction, it follows that there exists a fuzzy $\alpha$-open set $\rho \leq X$ containing $x_{\epsilon}$ such that $\rho \leq F^{+}(\mu)$. Since $\left(x_{\epsilon_{\alpha}}^{\alpha}\right) \alpha$-converges to $x_{\epsilon}$, it follows that there exists an index $\alpha_{o} \in J$ such that $\left(x_{\epsilon_{\alpha}}^{\alpha}\right) \in \rho$ for all $\alpha \geq \alpha_{o}$ from here, we obtain that $x_{\epsilon_{\alpha}}^{\alpha} \in \rho \leq F^{+}(\mu)$ for all $\alpha \geq \alpha_{o}$. Thus the net $\left(x_{\epsilon_{\alpha}}^{\alpha}\right)$ is eventually in $F^{+}(\mu)$.

(v) $\Rightarrow(\mathrm{i})$. Suppose that is not true. There exists a point $x_{\epsilon}$ and a fuzzy open set $\mu$ with $x_{\epsilon} \in F^{+}(\mu)$ such that $\rho \not \leq F^{+}(\mu)$ for each fuzzy $\alpha$-open set $\rho \leq X$ containing $x_{\epsilon}$. Let $x_{\epsilon_{\rho}} \in \rho$ and $x_{\epsilon} \notin F^{+}(\mu)$ for each fuzzy $\alpha$-open set $\rho \leq X$ containing $x_{\epsilon}$. Then for the $\alpha$-neighborhood net $\left(x_{\epsilon_{\rho}}\right), x_{\epsilon_{\rho}} \rightarrow_{\alpha} x_{\epsilon}$, but $\left(x_{\epsilon_{\rho}}\right)$ is not eventually in $F^{+}(\mu)$. This is a contradiction. Thus, $F$ is an upper fuzzy $\alpha$-continuous multifunction.

Remark 1. For a fuzzy multifunction $F: X \rightarrow Y$ from a fuzzy topological $(X, \tau)$ to a fuzzy topological space $(Y, v)$, the following implication holds:

Upper fuzzy continuous $\Longrightarrow$ Upper fuzzy $\alpha$-continuous.

The following example show that the reverse need not be true.

Example 1. Let $X=\{x, y\}$ with topologies $\tau=\{X, \phi, \mu\}$ and $v=\{X, \phi, \rho\}$, where the fuzzy sets $\mu, \rho$ are defined as:

$$
\begin{array}{cc}
\mu(x)=0.3, & \mu(y)=0.6 \\
\rho(x)=0.7, & \rho(y)=0.4
\end{array}
$$

A fuzzy multifunction $F:(X, \tau) \rightarrow(Y, \nu)$ given by $x_{\epsilon} \rightarrow F\left(x_{\epsilon}\right)=\left\{x_{\epsilon}\right\}$ is upper $\alpha$-continuous, but it is not upper continuous.

The following theorem states some characterizations of a lower fuzzy $\alpha$-continuous multifunction.

Theorem 2. Let $F: X \rightarrow Y$ be a fuzzy multifunction from a fuzzy topological $(X, \tau)$ to a fuzzy topological space $(Y, v)$. Then the following statements are equivalent.

(i) $F$ is lower fuzzy $\alpha$-continuous.

(ii) For each $x_{\epsilon} \in X$ and for each fuzzy open set $\mu$ such that $x_{\epsilon} \in F^{-}(\mu)$ there exists a fuzzy $\alpha$-open set $\rho$ containing $x_{\epsilon}$ such that $\rho \leq F^{-}(\mu)$.

(iii) $F^{-}(\mu)$ is a fuzzy $\alpha$-open set for any fuzzy open set $\mu \leq Y$, 
(iv) $F^{+}(\mu)$ is a fuzzy $\alpha$-closed set for any fuzzy open set $\mu \leq Y$,

(v) For each $x_{\epsilon} \in X$ and for each net $\left(x_{\epsilon_{\alpha}}^{\alpha}\right)$ which $\alpha$-converges to $x_{\epsilon}$ in $X$ and for each fuzzy open set $\mu \leq Y$ such that $x_{\epsilon} \in F^{-}(\mu)$, the net ( $\left.x_{\epsilon_{\alpha}}^{\alpha}\right)$ is eventually in $F^{-}(\mu)$.

Proof. It can be obtained similarly as Theorem 1.

Theorem 3. Let $F: X \rightarrow Y$ be a fuzzy multifunction from a fuzzy topological $(X, \tau)$ to a fuzzy topological space $(Y, v)$ and let $F(X)$ be endowed with subspace fuzzy topology. If $F$ is an upper fuzzy $\alpha$-continuous multifunction, then $F: X \rightarrow$ $F(X)$ is an upper fuzzy $\alpha$-continuous multifunction.

Proof. Since $F$ is an upper fuzzy $\alpha$-continuous, $F(X \wedge F(X))=F^{+}(\mu) \wedge$ $\wedge F^{+}(F(X))=F^{+}(\mu)$ is fuzzy $\alpha$-open for each fuzzy open subset $\mu$ of $Y$. Hence $F: X \rightarrow F(X)$ is an upper fuzzy $\alpha$-continuous multifunction.

Definition 4. Suppose that $(X, \tau),(Y, v)$ and $(Z, \omega)$ are fuzzy topological spaces. It is known that if $F_{1}: X \rightarrow Y$ and $F_{2}: Y \rightarrow Z$ are fuzzy multifunctions, then the fuzzy multifunction $F_{1} \circ F_{2}: X \rightarrow Z$ is defined by $\left(F_{1} \circ F_{2}\right)\left(x_{\epsilon}\right)=F_{2}\left(F_{1}\left(x_{\epsilon}\right)\right)$ for each $x_{\epsilon} \in X$.

Theorem 4. Let $(X, \tau),(Y, v)$ and $(Z, \omega)$ be fuzzy topological space and let $F$ : $X \rightarrow Y$ and $G: Y \rightarrow Z$ be fuzzy multifunction. If $F: X \rightarrow Y$ is an upper (lower) fuzzy continuous multifunction and $G: Y \rightarrow Z$ is an upper (lower) fuzzy $\alpha$-continuous multifunction. Then $G$ o $F: X \rightarrow Z$ is an upper (lower) fuzzy $\alpha$-continuous multifunction.

Proof. Let $\lambda \leq Z$ be any fuzzy open set. From the definition of $G$ o $F$, we have ( $G$ o $F)^{+}(\lambda)=F^{+}\left(G^{+}(\lambda)\right)\left((G \circ F)^{-}(\lambda)=F^{-}\left(G^{-}(\lambda)\right)\right)$, since $G$ is an upper (lower) fuzzy $\alpha$-continuous, it follows that $G^{+}(\lambda)\left(G^{-}(\lambda)\right)$ is a fuzzy open set. Since $F$ is an upper (lower) fuzzy continuous, it follows that $F^{+}\left(G^{+}(\lambda)\right)\left(F^{-}\left(G^{-}(\lambda)\right)\right)$ is a fuzzy $\alpha$-open set, this shows that $G$ o $F$ is an upper (lower) fuzzy $\alpha$-continuous.

Theorem 5. Let $F: X \rightarrow Y$ be a fuzzy multifunction from a fuzzy topological $(X, \tau)$ to a fuzzy topological space $(Y, \nu)$. If $F$ is a lower(upper) fuzzy $\alpha$-continuous multifunction and $\mu \leq X$ is a fuzzy set, then the restriction multifunction $\left.F\right|_{\mu}: \mu \rightarrow Y$ is an lower (upper) fuzzy $\alpha$-continuous multifunction.

Proof. Suppose that $\beta \leq Y$ is a fuzzy open set. Let $x_{\epsilon} \in \mu$ and let $\left.x_{\epsilon} \in F^{-}\right|_{\mu}(\beta)$. Since $F$ is a lower fuzzy $\alpha$-continuous multifunction, if follows that there exists a fuzzy open set $x_{\epsilon} \in \rho$ such that $\rho \leq F^{-}(\beta)$. From here we obtain that $x_{\epsilon} \in \rho \wedge \mu$ and $\rho \wedge \mu \leq\left. F\right|_{\mu}(\beta)$. Thus, we show that the restriction multifunction $\left.F\right|_{\mu}$ is lower fuzzy $\alpha$-continuous multifunction.

The proof for the case of the upper fuzzy $\alpha$-continuity of the multifunction $\left.F\right|_{\mu}$ is similar to the above. 
Theorem 6. Let $F: X \rightarrow Y$ be a fuzzy multifunction from a fuzzy topological $(X, \tau)$ to a fuzzy topological space $(Y, v)$, let $\left\{\lambda_{\gamma}: \gamma \in \Phi\right\}$ be a fuzzy open cover of $X$. If the restriction multifunction $F_{\gamma}=F_{\lambda_{\gamma}}$ is lower (upper) fuzzy $\alpha$-continuous multifunction for each $\gamma \in \Phi$, then $F$ is lower (upper) fuzzy $\alpha$-continuous multifunction.

Proof. Let $\mu \leq Y$ be any fuzzy open set. Since $F_{\gamma}$ is lower fuzzy $\alpha$-continuous for each $\gamma$, we know that $F_{\gamma}^{-}(\mu) \leq i n t_{\lambda_{\gamma}}\left(F_{\gamma}^{-}(\mu)\right)$ and from here $F^{-}(\mu) \wedge \lambda_{\gamma} \leq$ $\operatorname{int}_{\lambda_{\gamma}}\left(F^{-}(\mu) \wedge \lambda_{\gamma}\right)$ and $F^{-}(\mu) \wedge \lambda_{\gamma} \leq \operatorname{int}\left(F^{-}(\mu)\right) \wedge \lambda_{\gamma}$. Since $\left\{\lambda_{\gamma}: \gamma \in \Phi\right\}$ is a fuzzy open cover of $X$. It follows that $F^{-}(\mu) \leq \operatorname{int}\left(F^{-}(\mu)\right)$. Thus, we obtain that $F$ is lower(upper) fuzzy $\alpha$-continuous multifunction.

The proof of the upper fuzzy $\alpha$-continuity of $F$ is similar to the above.

Definition 5. Suppose that $F: X \rightarrow Y$ is a fuzzy multifunction from a fuzzy topological space $X$ to a fuzzy topological space $Y$. The fuzzy graph multifunction $G_{F}: X \rightarrow X \times Y$ of $F$ is defined as $G_{F}\left(x_{\epsilon}\right)=\left\{x_{\epsilon}\right\} \times F\left(x_{\epsilon}\right)$.

Theorem 7. Let $F: X \rightarrow Y$ be a fuzzy multifunction from a fuzzy topological $(X, \tau)$ to a fuzzy topological space $(Y, \nu)$. If the graph function of $F$ is lower(upper) fuzzy $\alpha$-continuous multifunction, then $F$ is lower(upper) fuzzy $\alpha$-continuous multifunction.

Proof. For the fuzzy sets $\beta \leq X, \eta \leq Y$, we take

$$
(\beta \times \eta)(z, y)= \begin{cases}0 & \text { if } z \notin \beta \\ \eta(y) & \text { if } z \in \beta\end{cases}
$$

Let $x_{\epsilon} \in X$ and let $\mu \in Y$ be a fuzzy open set such that $x_{\epsilon} \in F^{-}(\mu)$. We obtain that $x_{\epsilon} \in G_{F}^{-}(X \times \mu)$ and $X \times \mu$ is a fuzzy open set. Since fuzzy graph multifunction $G_{F}$ is lower fuzzy $\alpha$-continuous, it follows that there exists a fuzzy $\alpha$-open set $\rho \leq X$ containing $x_{\epsilon}$ such that $\rho \leq G_{F}^{-}(X \times \mu)$. From here, we obtain that $\rho \leq F^{-}(\mu)$. Thus, $F$ is lower fuzzy $\alpha$-continuous multifunction.

The proof of the upper fuzzy $\alpha$-continuity of $F$ is similar to the above.

Theorem 8. Suppose that $(X, \tau)$ and $\left(X_{\alpha}, \tau_{\alpha}\right)$ are fuzzy topological space where $\alpha \in J$. Let $F: X \rightarrow \prod_{\alpha \in J} X_{\alpha}$ be a fuzzy multifunction from $X$ to the product space $\prod_{\alpha \in J} X_{\alpha}$ and let $P_{\alpha}: \prod_{\alpha \in J} X_{\alpha} \rightarrow X_{\alpha}$ be the projection multifunction for each $\alpha \in J$ which is defined by $P_{\alpha}\left(\left(x_{\alpha}\right)\right)=\left\{x_{\alpha}\right\}$. If $F$ is an upper (lower) fuzzy $\alpha$-continuous multifunction, then $P_{\alpha} \circ F$ is an upper (lower) fuzzy $\alpha$-continuous multifunction for each $\alpha \in J$.

Proof. Take any $\alpha_{o} \in J$. Let $\mu_{\alpha_{o}}$ be a fuzzy open set in $\left(X_{\alpha}, \tau_{\alpha}\right)$. Then $\left(P_{\alpha_{o}}\right.$ o $F)^{+}\left(\mu_{\alpha_{o}}\right)=F^{+}\left(P_{\alpha_{o}}^{+}\left(\mu_{\alpha_{o}}\right)\right)=F^{+}\left(\mu_{\alpha_{o}} \times \prod_{\alpha \neq \alpha_{o}} X_{\alpha}\right)$ (resp., $\left(P_{\alpha_{o}} \text { o } F\right)^{-}\left(\mu_{\alpha_{o}}\right)=$ $\left.F^{-}\left(P_{\alpha_{o}}^{-}\left(\mu_{\alpha_{o}}\right)\right)=F^{-}\left(\mu_{\alpha_{o}} \times \prod_{\alpha \neq \alpha_{o}} X_{\alpha}\right)\right)$. 
Since $F$ is upper (lower) fuzzy $\alpha$-continuous multifunction and since $\mu_{\alpha_{o}} \times \prod_{\alpha \neq \alpha_{o}} X_{\alpha}$ is a fuzzy open set, it follows that $F^{+}\left(\mu_{\alpha_{o}} \times \prod_{\alpha \neq \alpha_{o}} X_{\alpha}\right)$ (resp., $\left.F^{-}\left(\mu_{\alpha_{o}} \times \prod_{\alpha \neq \alpha_{o}} X_{\alpha}\right)\right)$ is fuzzy $\alpha$ - open in $(X, \tau)$. It shows that $P_{\alpha_{o}} \circ F$ is upper (lower) fuzzy $\alpha$-continuous multifunction.

Hence, we obtain that $P_{\alpha}$ o $F$ is an upper (lower) fuzzy $\alpha$-continuous multi function for each $\alpha \in J$.

Theorem 9. Suppose that for each $\alpha \in J,\left(X_{\alpha}, \tau_{\alpha}\right)$ and $\left(Y_{\alpha}, v_{\alpha}\right)$ are fuzzy topological spaces. Let $F_{\alpha}: X_{\alpha} \rightarrow Y_{\alpha}$ be a fuzzy multifunction for each $\alpha \in J$ and let $F: \prod_{\alpha \in J} X_{\alpha} \rightarrow \prod_{\alpha \in J} Y_{\alpha}$ be defined by $F\left(\left(x_{\alpha}\right)\right)=\prod_{\alpha \in J} F_{\alpha}\left(x_{\alpha}\right)$ from the product space $\prod_{\alpha \in J} X_{\alpha}$ to product space $\prod_{\alpha \in J} Y_{\alpha}$. If $F$ is an upper (lower) fuzzy $\alpha$ continuous multifunction, then each $F_{\alpha}$ is an upper (lower) fuzzy $\alpha$-continuous multifunction for each $\alpha \in J$.

Proof. Let $\mu_{\alpha} \leq Y_{\alpha}$ be a fuzzy open set. Then $\mu_{\alpha} \times \prod_{\alpha \neq \beta} Y_{\beta}$ is a fuzzy open set. Since $F$ is an upper (lower) fuzzy $\alpha$-continuous multifunction, it follows that $F^{+}\left(\mu_{\alpha} \times \prod_{\alpha \neq \beta} Y_{\beta}\right)=F^{+}\left(\mu_{\alpha}\right) \times \prod_{\alpha \neq \beta} X_{\beta},\left(F^{-}\left(\mu_{\alpha} \times \prod_{\alpha \neq \beta} Y_{\beta}\right)=F^{-}\left(\mu_{\alpha}\right) \times\right.$ $\left.\prod_{\alpha \neq \beta} X_{\beta}\right)$ is a fuzzy $\alpha$-open set. Consequently, we obtain that $F^{+}\left(\mu_{\alpha}\right)\left(F^{-}\left(\mu_{\alpha}\right)\right)$ is a fuzzy $\alpha$-open set. Thus, we show that $F_{\alpha}$ is an upper (lower) fuzzy $\alpha$-continuous multifunction.

Theorem 10. Suppose that $\left(X_{1}, \tau_{1}\right),\left(X_{2}, \tau_{2}\right),\left(Y_{1}, v_{1}\right)$ and $\left(Y_{2}, v_{2}\right)$ are fuzzy topological spaces and $F_{1}: X_{1} \rightarrow Y_{1}, F_{2}: X_{2} \rightarrow Y_{2}$ are fuzzy multifunctions and suppose that if $\eta \times \beta$ is fuzzy $\alpha$-open set then $\eta$ and $\beta$ are fuzzy $\alpha$-open sets for any fuzzy sets $\eta \leq Y_{1}, \beta \leq Y_{2}$. Let $F_{1} \times F_{2}: X_{1} \times X_{2} \rightarrow Y_{1} \times Y_{2}$ be a fuzzy multifunction which is defined by $\left(F_{1} \times F_{2}\right)\left(x_{\epsilon}, y_{\nu}\right)=F_{1}\left(x_{\epsilon}\right) \times F_{2}\left(y_{\nu}\right)$. If $F_{1} \times F_{2}$ is an upper (lower) fuzzy $\alpha$-continuous multifunction, then $F_{1}$ and $F_{2}$ are upper (lower) fuzzy $\alpha$-continuous multifunctions.

Proof. We know that $\left(\mu^{*} \times \beta^{*}\right)\left(x_{\epsilon}, y_{\nu}\right)=\min \left\{\mu^{*}(x), \beta^{*}(y)\right\}$ for any fuzzy sets $\mu^{*}, \beta^{*}$ and for any fuzzy point $x_{\epsilon}, y_{\nu}$.

Let $\mu \times \beta \leq Y_{1} \times Y_{2}$ be a fuzzy open set. It known that $\left(F_{1} \times F_{2}\right)^{+}(\mu \times \beta)=$ $F_{1}^{+}(\mu) \times F_{2}^{+}(\beta)$. Since $F_{1} \times F_{2}$ is an upper fuzzy $\alpha$-continuous multifunction, it follows that $F_{1}^{+}(\mu) \times F_{2}^{+}(\beta)$ is a fuzzy $\alpha$-open set. From here, $F_{1}^{+}(\mu)$ and $F_{2}^{+}(\beta)$ are fuzzy $\alpha$-open sets. Hence, it is obtain that $F_{1}$ and $F_{2}$ are upper fuzzy $\alpha$-continuous multifunctions.

The proof of the lower fuzzy $\alpha$-continuity of the multifunctions $F_{1}$ and $F_{2}$ is similar to the above.

Theorem 11. Suppose that $(X, \tau),(Y, v)$ and $(Z, \omega)$ are fuzzy topological spaces and $F_{1}: X \rightarrow Y, F_{2}: X \rightarrow Z$ are fuzzy multifunction and suppose that if $\eta \times \beta$ is a fuzzy $\alpha$-open set, then $\eta$ and $\beta$ are fuzzy $\alpha$-open sets for any fuzzy sets $\eta \leq$ $Y, \beta \leq Z$. Let $F_{1} \times F_{2}: X \rightarrow Y \times Z$ be a fuzzy multifunction which is defined by 
$\left(F_{1} \times F_{2}\right)\left(x_{\epsilon}\right)=F_{1}\left(x_{\epsilon}\right) \times F_{2}\left(x_{\epsilon}\right)$. If $F_{1} \times F_{2}$ is an upper (lower) fuzzy $\alpha$-continuous multifunction, then $F_{1}$ and $F_{2}$ are upper (lower) fuzzy $\alpha$-continuous multifunctions.

Proof. Let $x_{\epsilon} \in X$ and let $\mu \leq Y, \beta \leq Z$ be fuzzy $\alpha$-open sets such that $x_{\epsilon} \in$ $F_{1}^{+}(\mu)$ and $x_{\epsilon} \in F_{2}^{+}(\beta)$. Then we obtain that $F_{1}\left(x_{\epsilon}\right) \leq \mu$ and $F_{2}\left(x_{\epsilon}\right) \leq \beta$ and from here, $F_{1}\left(x_{\epsilon}\right) \times F_{2}\left(x_{\epsilon}\right)=\left(F_{1} \times F_{2}\right)\left(x_{\epsilon}\right) \leq \mu \times \beta$. We have $x_{\epsilon} \in\left(F_{1} \times F_{2}\right)^{+}(\mu \times \beta)$. Since $F_{1} \times F_{2}$ is an upper fuzzy $\alpha$-continuous multifunction, it follows that there exist a fuzzy $\alpha$-open set $\rho$ containing $x_{\epsilon}$ such that $\rho \leq\left(F_{1} \times F_{2}\right)^{+}(\mu \times \beta)$. We obtain that $\rho \leq F_{1}^{+}(\mu)$ and $\rho \leq F_{2}^{+}(\beta)$. Thus we obtain that $F_{1}$ and $F_{2}$ are fuzzy $\alpha$-continuous multifunctions.

The proof of the lower fuzzy $\alpha$-continuity of the multifunctions $F_{1}$ and $F_{2}$ is similar to the above.

Lemma 1 ([2]). A fuzzy set in fuzzy topological space $X$ is a fuzzy $\alpha$-open set if and only if it is fuzzy semiopen and fuzzy preopen.

Theorem 12. Let $F: X \rightarrow Y$ be a fuzzy multifunction from a fuzzy topological $(X, \tau)$ to a fuzzy topological space $(Y, \nu)$. Then $F$ is an upper fuzzy $\alpha$-continuous if and only if it is an upper fuzzy semicontinuous and upper fuzzy precontinuous.

Proof. Let $F$ be upper fuzzy semicontinuous and upper fuzzy precontinuous, and let $\mu$ be a fuzzy open set in $Y$. Then $F^{+}(\mu)$ is fuzzy semiopen and fuzzy preopen, it follows from lemma 1 that $F^{+}(\mu)$ is a fuzzy $\alpha$-open set, and hence $F$ is an upper fuzzy $\alpha$-continuous multifunction. The converse is immediate.

Theorem 13. Let $F: X \rightarrow Y$ be a fuzzy multifunction from a fuzzy topological space $(X, \tau)$ to a fuzzy topological space $(Y, v)$. Then $F$ is a lower fuzzy $\alpha$-continuous if and only if it is lower fuzzy semicontinuous and lower fuzzy precontinuous.

Proof. Similar to that of Theorem 12 and is omitted.

\section{REFERENCES}

[1] K. K. Azad, "On fuzzy semicontinuity, fuzzy almost continuity and fuzzy weakly continuity," $J$. Math. Anal. Appl., vol. 82, no. 1, pp. 14-32, 1981.

[2] A. S. Bin Shahna, "On fuzzy strong semicontinuity and fuzzy precontinuity," Fuzzy Sets and Systems, vol. 44, no. 2, pp. 303-308, 1991.

[3] C. L. Chang, "Fuzzy topological spaces," J. Math. Anal. Appl., vol. 24, pp. 182-190, 1968.

[4] A. S. Mashhour, M. E. Abd El-Monsef, and S. N. El-Deep, "On precontinuous and weak precontinuous mappings," Proc. Math. Phys. Soc. Egypt, vol. 53, pp. 47-53, 1982.

[5] M. N. Mukherjee and S. Malakar, "On almost continuous and weakly continuous fuzzy multifunctions," Fuzzy Sets and Systems, vol. 41, no. 1, pp. 113-125, 1991.

[6] T. Neubrunn, Strongly quasi-continuous multivalued mappings, ser. Research and Exposition in Mathematics. Berlin: Heldermann, 1988, vol. 16.

[7] O. Njåstad, “On some classes of nearly open sets," Pacific J. Math., vol. 15, pp. 961-970, 1965.

[8] N. S. Papageorgiou, "Fuzzy topology and fuzzy multifunctions," J. Math. Anal. Appl., vol. 109, no. 2, pp. 397-425, 1985. 
[9] V. Popa and T. Noiri, "On upper and lower $\alpha$-continuous multifunctions," Math. Slovaca, vol. 43, no. 4, pp. 477-491, 1993.

[10] P. M. Pu and Y. M. Liu, "Fuzzy topology. i. neighborhood structure of a fuzzy point and mooresmith convergence," J. Math. Anal. Appl., vol. 76, no. 2, pp. 571-599, 1980.

[11] P.-M. Pu and Y. M. Liu, "Fuzzy topology. ii. product and quotient spaces," J. Math. Anal. Appl., vol. 77, no. 1, pp. 20-37, 1980.

[12] L. A. Zadeh, "Fuzzy sets," Inform. and Control, vol. 8, pp. 338-353, 1965.

Authors' addresses

Khaled M. A. Al-Hamadi

Dept. of Mathematics, University of Pune

E-mail address: abusuliman88@yahoo.com

S. B. Nimse

Dept. of Mathematics, University of Pune

E-mail address: nacasca@rediffmail.com 